2020, Instituto Mexicano de Tecnología del Agua

Tecnología y

Ciencias $₫$ Agua
Open Access bajo la licencia CC BY-NC-SA 4.0 (https://creativecommons.org/licenses/by-nc$\mathrm{sa} / 4.0 /$ )

DOI: $10.24850 / \mathrm{j}$-tyca-2020-03-08

Artículos

\title{
Remineralización de agua osmotizada mediante un contactor de calcita de flujo continuo ascendente
}

\section{Remineralization of osmotic water using a continuous upflow calcite contactor}

Iván Emmanuel Villegas-Mendoza1 ${ }^{1}$, ORCID: https://orcid.org/0000-00021367-6558

Martín Piña-Soberanis², ORCID: https://orcid.org/0000-0002-8342-097X Angélica Julieta Alvillo-Rivera ${ }^{3}$, ORCID: https://orcid.org/0000-00026759-5227

Carlos David Silva-Luna4, ORCID: https://orcid.org/0000-0003-36458120

José de Jesús Linares-Ocampo 5

${ }^{1}$ Instituto Mexicano de Tecnología del Agua, Jiutepec, Morelos, México, ivan_villegas@tlaloc.imta.mx

2Instituto Mexicano de Tecnología del Agua, Jiutepec, Morelos, México, mpina@tlaloc.imta.mx

3Instituto Mexicano de Tecnología del Agua, Jiutepec, Morelos, México, julieta.alvillo@posgrado.imta.edu.mx 


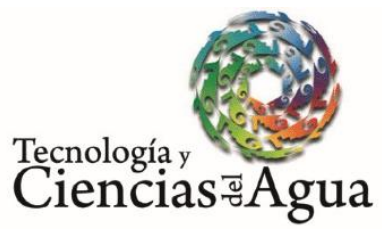

2020, Instituto Mexicano de Tecnología del Agua

Open Access bajo la licencia CC BY-NC-SA 4.0 (https://creativecommons.org/licenses/by-ncsa/4.0/)

${ }^{4}$ Instituto Mexicano de Tecnología del Agua, Jiutepec, Morelos, México, carlos_silva@tlaloc.imta.mx

${ }^{5}$ Instituto Mexicano de Tecnología del Agua, Jiutepec, Morelos, México, j.linareso@hotmail.com

Autor para correspondencia: Iván Emmanuel Villegas-Mendoza, ivan_villegas@tlaloc.imta.mx

\section{Resumen}

En este trabajo se evaluó la influencia de la velocidad de flujo, tiempo de contacto y $\mathrm{pH}$ en un proceso de remineralización de agua osmotizada mediante un contactor piloto de calcita a flujo continuo para la producción de agua potable. Se utilizó agua procedente de una planta desaladora de agua de mar en México, la cual carece de postratamiento (remineralización). Las pruebas experimentales se llevaron a cabo mediante el diseño y la construcción de un contactor de calcita de altura variable y ajuste de $\mathrm{pH}$ del influente con la adición de ácido sulfúrico. Los rangos de estudio evaluados fueron los siguientes: velocidad de flujo, 4$8 \mathrm{~m} \mathrm{~h}^{-1}$; tiempo de residencia hidráulico, 10-30 min, y pH de 3 a 6 . Se implementó un diseño experimental $2^{k}$, en el que la variable de respuesta fue el índice de saturación de Langelier. Los resultados indicaron que de los tres parámetros de diseño evaluados el único que tuvo un efecto estadísticamente significativo fue el $\mathrm{pH}$ del influente, siendo el óptimo igual a tres. La calidad del agua remineralizada cumplió con la regulación israelí de contenido mineral para agua potable. 


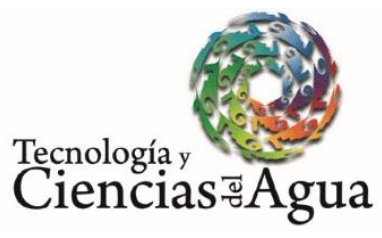

2020, Instituto Mexicano de Tecnología del Agua

Open Access bajo la licencia CC BY-NC-SA 4.0 (https://creativecommons.org/licenses/by-ncsa/4.0/)

Los resultados mostraron la conveniencia de diseñar contactores de calcita con velocidad de flujo de $8 \mathrm{~m} \mathrm{~h}^{-1}$ y 10 minutos de tiempo de residencia hidráulico, con el objetivo de disminuir los costos de inversión. Estas condiciones permiten diseñar contactores más compactos sin incidir en la eficiencia del proceso. El costo de operación del proceso se calculó en $\$ 0.04$ USD $\mathrm{m}^{-3}$.

Palabras clave: remineralización, agua osmotizada, contactor de calcita.

\section{Abstract}

In this work, the influence of the flow velocity, contact time and $\mathrm{pH}$ in the process of remineralization of osmotized water was evaluated by means a pilot contactor of calcite to continuous flow to produce drinking water. Water from a Mexican seawater desalination plant that lacks posttreatment (remineralization) was used. The experimental tests were carried out by designing and building a contactor of calcite of variable height adjusting $\mathrm{pH}$ of the influent by adding sulfuric acid.

The study ranges evaluated the following: flow rate, 4-8 $\mathrm{m} \mathrm{h}^{-1}$; hydraulic residence time, $10-30 \mathrm{~min}$, and $\mathrm{pH} 3$ to 6 . An experimental $2^{\mathrm{k}}$ design was implemented, in which the response variable was the Langelier saturation index. The results showed for the three parameters evaluated, that only the $\mathrm{pH}$ of the influent had a statistical significance, with the optimum being $\mathrm{pH}=3$. The quality of the remineralized water complied with the Israeli regulation of mineral content for drinking water.

The results showed the desirability of designing calcite contactors with a flow velocity of $8 \mathrm{~m} \mathrm{~h}^{-1}$ and 10 minutes of hydraulic residence time, 


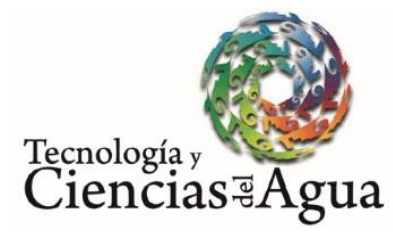

2020, Instituto Mexicano de Tecnología del Agua

Open Access bajo la licencia CC BY-NC-SA 4.0 (https://creativecommons.org/licenses/by-ncsa/4.0/)

to reduce investment costs. These conditions make it possible to design more compact contactors without affecting the efficiency of the process. The operating cost of the process was calculated at $\$ 0.04$ USD $\mathrm{m}^{-3}$.

Keywords: Remineralization, desalinated water, calcite contactor.

Recibido: $11 / 07 / 2018$

Aceptado: 16/09/2019

\section{Introducción}

Al igual que muchos países en el mundo, México comienza a enfrentarse con dificultades en el suministro de agua potable a la población debido a la sobreexplotación y contaminación de acuíferos. Para solventar dichos problemas, se ha estudiado el uso de tecnologías no convencionales para la potabilización del agua, como la desalinización del agua de mar. En la actualidad, México cuenta con 301 plantas municipales $\left(Q=2983 \mathrm{~L} \mathrm{~s}^{-1}\right)$, que utilizan como tecnología de tratamiento la ósmosis inversa (Conagua, 2015).

Los procesos de desalinización con ósmosis inversa se caracterizan por eliminar en gran medida los minerales y sales del agua de 


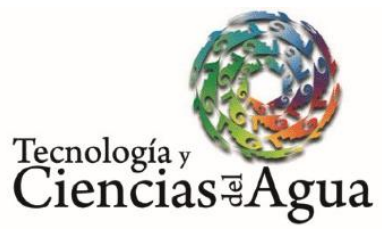

2020, Instituto Mexicano de Tecnología del Agua

Open Access bajo la licencia CC BY-NC-SA 4.0 (https://creativecommons.org/licenses/by-nc-

sa/4.0/)

alimentación, produciendo un agua no estable que puede causar problemas en la salud de la población que la bebe. Además, el agua osmotizada genera problemas de corrosión en las tuberías y/o estructuras auxiliares en los sistemas de distribución (Biyoune et al., 2017; Liang et al., 2013a; El-Azhar et al., 2012), por lo cual es indispensable contar con sistemas de postratamiento.

Los sistemas de postratamiento de agua osmotizada se enfocan a remineralizarla y estabilizarla; y se han convertido en una parte integral en las plantas desalinizadoras. El propósito más importante de la remineralización es producir agua sin tendencia a provocar corrosión de partes metálicas y la desintegración de componentes de concreto en los sistemas de distribución de agua (Bergman, 2007); asimismo, la corrosión de las tuberías se asocia con riesgos a la salud por la liberación no deseable de iones metálicos (Fe, Pb, $\mathrm{Cu}$ y $\mathrm{Zn}$ ) (Birnhack, Voutchkov, \& Lahay, 2011), y compuestos como $\mathrm{Fe}_{3} \mathrm{O}_{4}$, geotita y hematita (Liang et al., 2013b), los cuales son procesos que vuelven insalubre el agua.

Se ha reportado que beber agua desmineralizada o un líquido hipotónico puede ser perjudicial para la salud (Vingerhoeds et al., 2016), ya que llega a provocar síntomas como cansancio, debilidad, dolor de cabeza y edema cerebral; esto, derivado de la baja ingesta mineral (iones de Ca y Mg). En infantes se ha reportado ser causal de acidosis metabólica (Kozisek, Rosborg, Selinus, Ferrante \& Jovanovic, 2015). Además, se incrementan los costos de mantenimiento y disminuye la longevidad de los sistemas de distribución (Birnhack et al., 2011; Sarin, Snoeyink, Bebee, Kriven, \& Clement, 2001; Edwards, 2004; McNeill \& Edwards, 2001). 
Tecnología y

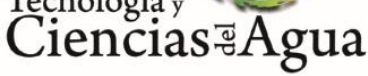

2020, Instituto Mexicano de Tecnología del Agua

Open Access bajo la licencia CC BY-NC-SA 4.0 (https://creativecommons.org/licenses/by-nc$\mathrm{sa} / 4.0 /$ )

Para la medición de la corrosividad del agua se utiliza principalmente el índice de saturación de Langelier (ISL) (Tavanpour, Noshadi, \& Navid, 2016; Awatif, Basim, \& Sura-Ali, 2014):

$$
I S L=p H a-p H s=p H a-((9.3+A+B)-(C+D))
$$

Donde:

$p H a=p H$ del agua.

$p H s=p H$ de saturación.

$A=\left(\log _{10}(\right.$ sólidos disueltos totales $\left.)-1\right) / 10$.

$B=-13.12 * \log _{10}\left(\right.$ temperatura del agua en $\left.{ }^{\circ} \mathrm{C}+273\right)+34.55$.

$C=\log _{10}\left[\mathrm{Ca}^{2+}\right.$ en $\mathrm{mg} \mathrm{L}^{-1}$ de $\left.\mathrm{CaCO}_{3}\right]-0.4$.

$D=\log _{10}\left[\right.$ alcalinidad en $\mathrm{mg} \mathrm{L}^{-1}$ de $\left.\mathrm{CaCO}_{3}\right]$.

Los valores negativos de ISL indican que el agua tiene una tendencia corrosiva; valores positivos señalan una tendencia incrustante; mientras que un valor de cero indica que el agua está en equilibrio químico (Pauta-Novillo \& Quezada-Sisalima, 2017; Tavanpour et al., 2016).

Israel es una superpotencia en desalinización de agua de mar. En 2015 se desalinizaron 600 millones de metros cúbicos, lo que equivale al $32 \%$ de toda el agua doméstica consumida en dicho país, y se espera que esta proporción incremente a 75\% en 2025 (Vasiliy, Garbera, \& Chenb, 2018). Por otra parte, las regulaciones israelís establecen que el agua para consumo humano debe tener un ISL $>0$. La Tabla 1 muestra otros 
Tecnología y

Ciencias $\stackrel{\unlhd}{\unlhd}$ Agua
2020, Instituto Mexicano de Tecnología del Agua

Open Access bajo la licencia CC BY-NC-SA 4.0 (https://creativecommons.org/licenses/by-nc-

$\mathrm{sa} / 4.0 /$ )

parámetros de contenido mineral que ha regulado el Ministerio de Salud Israelí para agua de mar potabilizada (Shemer, Hasson, \& Semiat, 2015).

Tabla 1. Regulación israelí para agua de mar potabilizada.

\begin{tabular}{|c|c|}
\hline Parámetro & Valor \\
\hline $\mathrm{pH}$ & $7.5-8.3$ \\
\hline Alcalinidad (mg L & \\
\hline Calcio (mg L-1 $\left.\mathrm{CaCO}_{3}\right)$ & $>80$ \\
\hline Dureza total $\left(\mathrm{mg} \mathrm{L}^{-1} \mathrm{CaCO}_{3}\right)$ & $80-120$ \\
\hline Turbiedad (UTN) & $<0.5$ \\
\hline ISL & $>0$ \\
\hline
\end{tabular}

La normatividad mexicana de agua potable (modificación del año 2000 a la NOM-127-SSA1-1994) (DOF, 1994) no regula el ISL, calcio ni alcalinidad; tampoco establece un límite mínimo permisible de dureza total, sin embargo, es importante tomar estos parámetros en cuenta al momento de proporcionar agua a la población debido a los diversos problemas de salud y a la red de suministro de agua que provoca la deficiencia de sales.

Las técnicas de remineralización pueden dividirse en tres grupos de tecnologías: (1) procesos que se basan en la adición directa de sustancias químicas que contienen calcio (p. ej., hidróxido de calcio, cloruro de calcio, bicarbonato de sodio) y magnesio (p. ej., cloruro de magnesio y sulfato de magnesio); (2) procesos en los que la remineralización se 03-08 


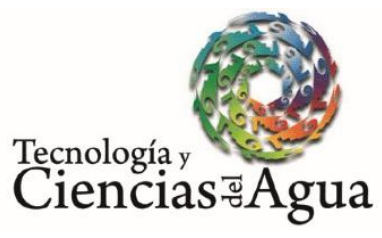

2020, Instituto Mexicano de Tecnología del Agua

Open Access bajo la licencia CC BY-NC-SA 4.0 (https://creativecommons.org/licenses/by-nc-

sa/4.0/)

realiza mediante la mezcla de agua desalinizada con una porción del agua que se utiliza para llevar a cabo la desalinización, o con otras fuentes de agua con elevado contenido de calcio y magnesio; y (3) procesos en los que el calcio y magnesio se agregan mediante la disolución de minerales como calcita (carbonato de calcio) (Voutchkov, 2011; Withers, 2005).

La disolución de calcita tiene como funciones principales el enriquecimiento del agua con minerales y el aumento de alcalinidad, dureza y pH (Sousi et al., 2018; Liang et al., 2013a). Algunas de las ventajas sobre los otros procesos de remineralización son:

- No adiciona iones no deseados, como sodio $\left(\mathrm{Na}^{+}\right)$, sulfato $\left(\mathrm{SO}_{4}{ }^{2-}\right)$ y cloruros $\left(\mathrm{Cl}^{-}\right)$, entre otros.

- No agrega turbidez al agua producto.

- Produce agua con elevada capacidad amortiguadora.

- Facilita la operación.

- No existe riesgo de una sobredosificación.

Este método es rutinariamente usado en regiones donde la calcita está disponible a un precio accesible, como Europa, Medio Oriente y los países del Golfo Pérsico (Lehmann, Birnhack, \& Lahav, 2013). En México existen importantes yacimientos de calcita blanca de alta pureza, por lo que es factible su implementación.

El proceso de disolución de calcita en agua se realiza a un pH ácido, en tanques empacados con calcita, donde se controla el $\mathrm{pH}$ del influente; estos equipos son llamados por lo común "contactores de calcita o filtros de calcita". La disolución de calcita resulta en el enriquecimiento del agua con iones de calcio y alcalinidad por bicarbonatos.

Tecnología y ciencias del agua, ISSN 2007-2422,11(3), 277-308. DOI: 10.24850/j-tyca-202003-08 


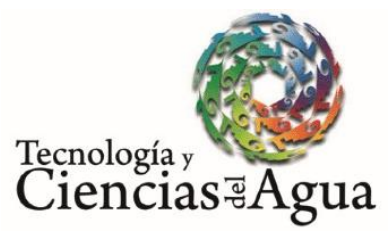

2020, Instituto Mexicano de Tecnología del Agua

Open Access bajo la licencia CC BY-NC-SA 4.0 (https://creativecommons.org/licenses/by-nc$\mathrm{sa} / 4.0 /$ )

En la práctica, la reducción de pH se lleva a cabo mediante la adición de un ácido fuerte $\left(\mathrm{H}_{2} \mathrm{SO}_{4}\right)$ o uno débil $\left(\mathrm{CO}_{2}\right)$. Cuando se utiliza ácido sulfúrico, en conjunto con calcita, ocurre una reacción (Ecuación (2)) en la que se forma dióxido de carbono y sulfato de calcio; mientras que en una reacción secundaria (Ecuación (3)) entre el dióxido de carbono y la calcita $\left(\mathrm{CaCO}_{3}\right)$ se forma bicarbonato de calcio. Las reacciones involucradas son las siguientes (Hernández-Suárez, 2010):

$$
\begin{gathered}
\mathrm{CaCO}_{3}+\mathrm{H}_{2} \mathrm{SO}_{4} \rightarrow \mathrm{CaSO}_{4}+\mathrm{H}_{2} \mathrm{O}+\mathrm{CO}_{2} \\
\mathrm{CaCO}_{3}+\mathrm{CO}_{2}+\mathrm{H}_{2} \mathrm{O} \rightarrow \mathrm{Ca}\left(\mathrm{HCO}_{3}\right)_{2}
\end{gathered}
$$

En resumen:

$$
2 \mathrm{CaCO}_{3}+\mathrm{H}_{2} \mathrm{SO}_{4}+\mathrm{H}_{2} \mathrm{O} \rightarrow \mathrm{Ca}\left(\mathrm{HCO}_{3}\right)_{2}+\mathrm{CaSO}_{4}
$$

Los contactores de calcita han sido utilizados por décadas, sin embargo existe poca información en la literatura científica sobre los parámetros de diseño imperativos de esta tecnología (Lehmann et al., 2013), los cuales inciden en el costo de inversión y operación. Los principales criterios de diseño son la velocidad de flujo, tiempo de residencia y $\mathrm{pH}$. Voutchkov (2011) señala que la velocidad del agua en los contactores de calcita debe ser de 4 a $8 \mathrm{~m} \mathrm{~h}^{-1}$; el tiempo de residencia hidráulico necesita oscilarentre 10 y 30 minutos, y el pH sermenor a 4.5. 


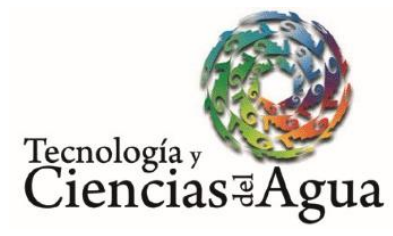

2020, Instituto Mexicano de Tecnología del Agua

Open Access bajo la licencia CC BY-NC-SA 4.0 (https://creativecommons.org/licenses/by-nc$\mathrm{sa} / 4.0 /$ )

Por otra parte, Hasson, Fine, Sagiv, Semiat y Shemer (2017) señalan que el tiempo de contacto oscila de 20 a 35 minutos y la velocidad de flujo de 5 a $15 \mathrm{~m} \mathrm{~h}^{-1}$. La literatura no indica el efecto que tienen estos parámetros en la eficiencia de remineralización. Además, los rangos son amplios y requieren mayor estudio para diseñar contactores eficientes a un bajo costo de inversión y operación.

El objetivo de este trabajo fue evaluar el efecto que tienen la velocidad de flujo, el tiempo de residencia hidráulico y el $\mathrm{pH}$ en el proceso de remineralización de agua osmotizada en un contactor de calcita a flujo continuo, así como encontrar los criterios de diseño/operación que permitan diseñar contactores de calcita eficientes para producir agua que cumpla con el contenido mineral que establece la regulación israelí de agua potable al menor costo de operación e inversión.

\section{Materiales y métodos}

\section{Etapa 1}


Tecnología y

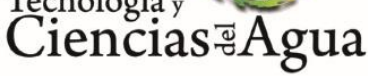

2020, Instituto Mexicano de Tecnología del Agua

Open Access bajo la licencia CC BY-NC-SA 4.0 (https://creativecommons.org/licenses/by-nc$\mathrm{sa} / 4.0 /$ )

La primera etapa se enfocó en evaluar el efecto que tienen la velocidad de flujo, el tiempo de residencia hidráulico y el $\mathrm{pH}$ en el proceso de remineralización de agua osmotizada en un contactor de calcita a flujo continuo, para lo cual se implementó un diseño de experimentos factorial $2^{k}$, específicamente un $2^{3}$, el cual consistió de 11 pruebas experimentales, cada una con una duración de dos horas. Cada factor se evaluó en tres niveles: bajo, alto y medio. La Tabla 2 muestra los niveles elegidos para cada uno de los factores sujetos a estudio. Los niveles abarcaron los valores altos y bajos recomendados por la literatura, a fin de evaluar en un amplio intervalo el efecto de cada uno de los factores.

Tabla 2. Niveles de los factores sujetos a estudio.

\begin{tabular}{|c|c|c|c|}
\hline Nivel & $\begin{array}{c}\text { Velocidad de } \\
\text { flujo }\left(\mathbf{m ~ h}^{-\mathbf{1}} \mathbf{)}\right.\end{array}$ & $\begin{array}{c}\text { Tiempo de } \\
\text { residencia } \\
\mathbf{( m i n )}\end{array}$ & $\mathbf{p H}$ \\
\hline Bajo & 4 & 10 & 3 \\
\hline Alto & 8 & 20 & 6 \\
\hline Central & 6 & 15 & 4.5 \\
\hline
\end{tabular}

La Tabla 3 muestra la matriz de experimentos, es decir, el orden en el que se llevaron a cabo las 11 pruebas y la combinación de factores en cada una de ellas. Se realizaron tres réplicas del punto central (pruebas 1,2 y 11) para obtener grados de libertad adicionales y lograr con ello una adecuada estimación del error del análisis de varianza, ya que éste 
Tecnología y

Ciencias $₫$ Agua
2020, Instituto Mexicano de Tecnología del Agua

Open Access bajo la licencia CC BY-NC-SA 4.0 (https://creativecommons.org/licenses/by-ncsa/4.0/)

debe contar con al menos ocho grados de libertad (Gutiérrez \& Salazar, 2012).

Tabla 3. Matriz experimental del diseño $2^{3}$ empleado.

\begin{tabular}{|c|c|c|c|}
\hline $\begin{array}{c}\text { Núm. de } \\
\text { prueba }\end{array}$ & $\mathbf{p H}$ & $\begin{array}{c}\text { Tiempo de } \\
\text { residencia (min) }\end{array}$ & $\begin{array}{c}\text { Velocidad de } \\
\text { flujo }\left(\mathbf{m ~ h}^{\mathbf{- 1}} \mathbf{)}\right.\end{array}$ \\
\hline 1 & 4.5 & 15 & 6 \\
\hline 2 & 4.5 & 15 & 6 \\
\hline 3 & 3 & 20 & 8 \\
\hline 4 & 6 & 10 & 4 \\
\hline 5 & 6 & 10 & 8 \\
\hline 6 & 3 & 10 & 4 \\
\hline 7 & 6 & 20 & 8 \\
\hline 8 & 6 & 20 & 8 \\
\hline 9 & 3 & 20 & 6 \\
\hline 10 & 3 & 10 & 45 \\
\hline 11 & 4.5 & 15 & \\
\hline
\end{tabular}

Etapa 2 


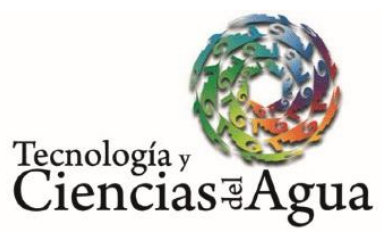

2020, Instituto Mexicano de Tecnología del Agua

Open Access bajo la licencia CC BY-NC-SA 4.0 (https://creativecommons.org/licenses/by-ncsa/4.0/)

La segunda etapa consistió en realizar una prueba bajo las mejores condiciones de diseño/operación encontradas en la etapa 1 durante 40 horas, tomando muestras cada dos horas para analizar los parámetros que se requieren para calcular el ISL y compararlos con la regulación israelí para agua potable remineralizada.

\section{Agua osmotizada}

El agua que se utilizó para realizar las pruebas fue el efluente de una planta desaladora de agua de mar en México que no cuenta con un sistema de remineralización (Figura 1). La capacidad de la planta es de 3 $456 \mathrm{~m}^{3} \mathrm{~d}^{-1}$ y produce un agua con un ISL de $-4.58 \pm 0.22$ (con tendencia a provocar corrosión severa), la cual se recolectó en un tanque de 1000 L, donde se adicionó, en su caso, ácido sulfúrico al 98\% (grado comercial) para obtener el pH requerido en cada prueba. Posteriormente, el agua se pasó a través de un contactor de calcita, utilizando una bomba peristáltica marca Cole-Parmer modelo 7591-22, equipada con un controlador de flujo, que permitió mantener estable la velocidad de flujo y el tiempo de contacto requeridos en cada prueba. 


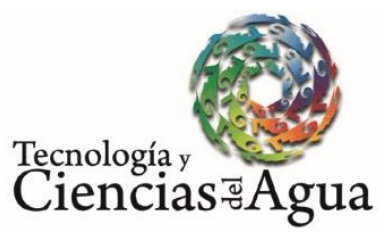

2020, Instituto Mexicano de Tecnología del Agua

Open Access bajo la licencia CC BY-NC-SA 4.0 (https://creativecommons.org/licenses/by-nc-

$\mathrm{sa} / 4.0 /$ )

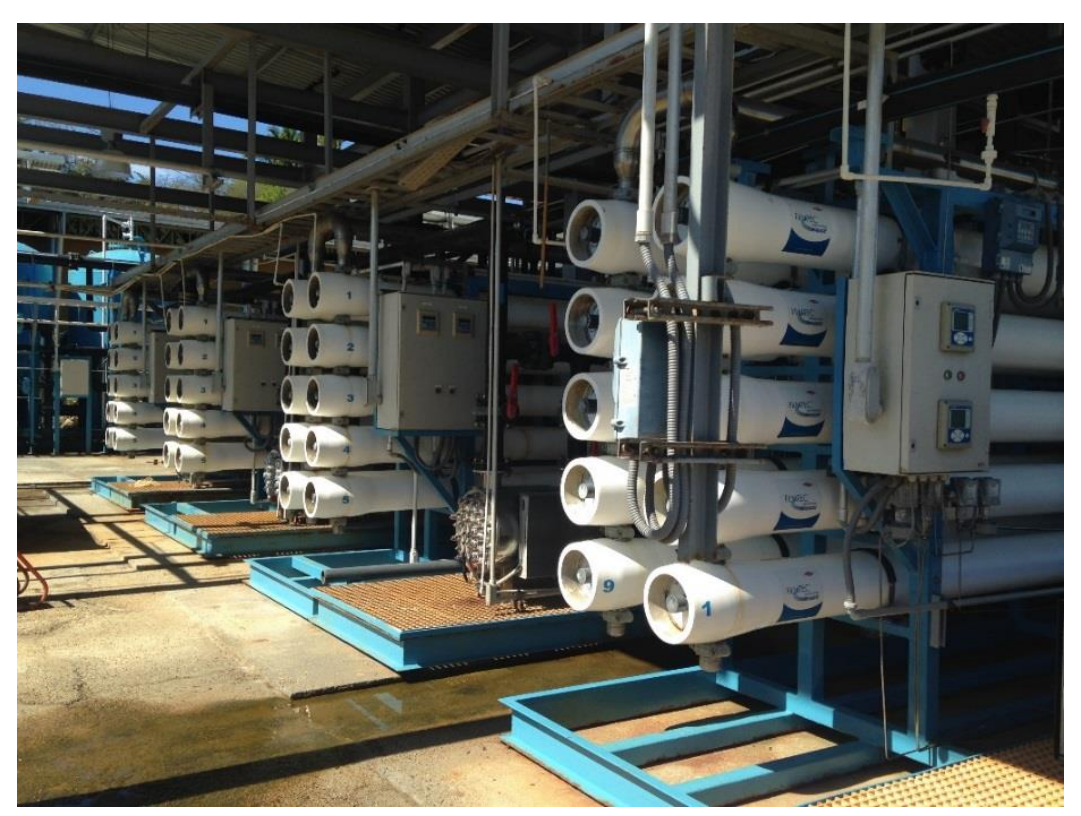

Figura 1. Planta desaladora de agua de mar.

\section{Contactor de calcita}

El material seleccionado para empacar el contactor fue calcita, ya que presenta menores costos (Voutchkov, 2011). El bicarbonato de sodio y el sulfato de calcio cuentan con baja solubilidad en el agua (Biyoune et al., 2017). Por otra parte, el carbonato de sodio tiende a producir depósitos no adherentes de $\mathrm{CaCO}_{3}$ (Biyoune et al., 2017; Withers, 2005); mientras que la cal hidratada no se disuelve fácilmente, lo que provoca obtener valores de turbiedad mayores a 5 NTU (Delion, Nauguin, \& Corsin, 2004). En cuanto al reactivo para la acidificación del agua, se eligió $\mathrm{H}_{2} \mathrm{SO}_{4}$ sobre 
2020, Instituto Mexicano de Tecnología del Agua

Tecnología y

Ciencias $\stackrel{\Xi}{\unlhd}$ Aua
Open Access bajo la licencia CC BY-NC-SA 4.0 (https://creativecommons.org/licenses/by-nc$\mathrm{sa} / 4.0 /$ )

$\mathrm{CO}_{2}$, por presentar mayores velocidades de reacción y menores costos (Shemer et al., 2015; Birnhack et al., 2011).

El contactor de calcita se operó a flujo ascendente y se construyó con material de acrílico. Se le colocó una placa de orificios en la parte inferior para distribuir de forma homogénea el flujo de agua. El contactor se empacó con $10 \mathrm{~cm}$ de material de soporte (grava sílica, de tamaño $\left.1 / 8 \times 1 / 16^{\prime \prime}\right)$. El área superficial de contacto fue de $63.6 \mathrm{~cm}^{2}$. Las cantidades adicionadas de calcita fueron de $10.92,7.8$ y $5.46 \mathrm{~kg}$ cuando se utilizaron tiempos de residencia de 20,15 y $10 \mathrm{~min}$, respectivamente. La calcita empleada fue de una pureza mayor al $98 \%$ y en forma granular, con diámetro efectivo de $1 \mathrm{~mm}$. Cada una de las pruebas tuvo una duración de cinco horas. El diagrama del experimento se muestra en la Figura 2.

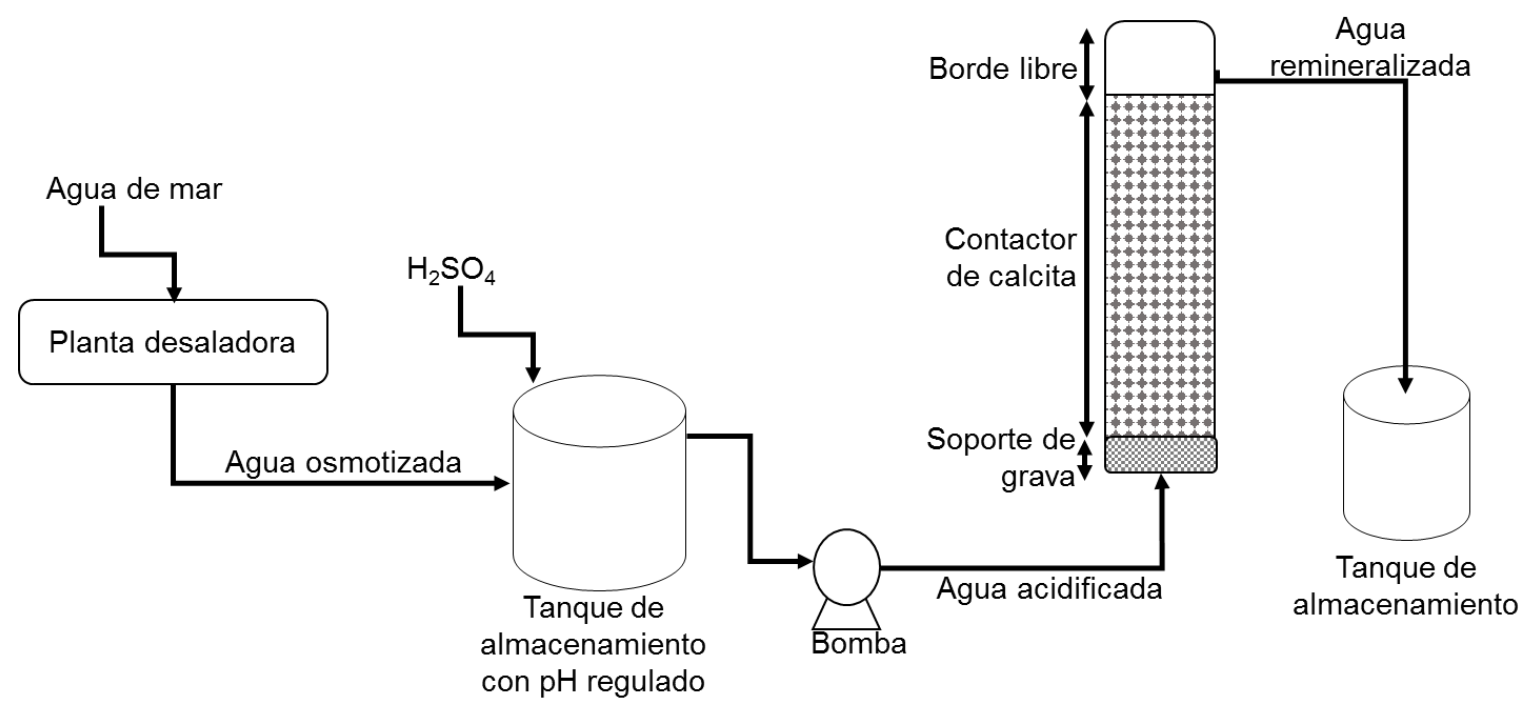

Figura 2. Diagrama del experimento. 
Teçnología y

Ciencias $\stackrel{\unlhd}{\unlhd}$ gua
2020, Instituto Mexicano de Tecnología del Agua

Open Access bajo la licencia CC BY-NC-SA 4.0 (https://creativecommons.org/licenses/by-nc$\mathrm{sa} / 4.0 /$ )

La variable de respuesta fue el índice de saturación de Langelier (ISL), el cual se calcula en función del pH, alcalinidad, sólidos disueltos totales, dureza total y temperatura, y es un indicador de la tendencia corrosiva o incrustativa del agua.

\section{Determinación de parámetros físico-químicos}

La determinación de $\mathrm{pH}$ se realizó utilizando un potenciómetro marca Orion Modelo 420a, mientras que los sólidos disueltos totales se midieron utilizando un conductímetro Orion Modelo 145. La concentración de alcalinidad, dureza total y sulfatos se midieron usando métodos colorimétricos, con un espectrofotómetro marca HACH modelo DR2800.

\section{Análisis estadístico}

Los resultados obtenidos en las pruebas experimentales se analizaron mediante el paquete de cómputo estadístico Statgraphics Centurión XV 
2020, Instituto Mexicano de Tecnología del Agua

Tecnología y

Ciencias $₫$ Agua
Open Access bajo la licencia CC BY-NC-SA 4.0 (https://creativecommons.org/licenses/by-nc$\mathrm{sa} / 4.0 /$ )

versión 15.2.06, del que se lograron análisis de varianza, gráficas de efectos principales, diagramas de Pareto y gráficas de superficie de respuesta, para la variable de respuesta analizada.

\section{Costo de operación}

El costo de operación del proceso de remineralización se calculó de acuerdo con la Ecuación (5):

$$
C O=C_{A} B_{A}+C_{c} C_{B}
$$

Donde:

$\mathrm{CO}=$ costo de operación del contactor $\left(\$ \mathrm{USD} \mathrm{\textrm {m } ^ { - 3 }}\right)$.

$\mathrm{C}_{\mathrm{A}}=$ consumo de ácido sulfúrico para obtener el pH óptimo $\left(\mathrm{L} \mathrm{m}^{-3}\right)$.

$\mathrm{B}_{\mathrm{A}}=$ costo de ácido sulfúrico ( $\$$ USD $\left.\mathrm{L}^{-1}\right)$.

$\mathrm{C}_{\mathrm{c}}=$ consumo de calcita $\left(\$ \mathrm{~kg} \mathrm{~m}^{-3}\right)$.

$\mathrm{B}_{\mathrm{A}}=$ costo de calcita $\left(\$ \mathrm{USD} \mathrm{kg}{ }^{-1}\right)$. 
Tecnología y

Ciencias $\approx$ Agua
2020, Instituto Mexicano de Tecnología del Agua

Open Access bajo la licencia CC BY-NC-SA 4.0 (https://creativecommons.org/licenses/by-nc$\mathrm{sa} / 4.0 /$ )

\section{Resultados y discusión}

\section{Etapa 1}

El análisis de varianza (ANOVA) de los resultados del diseño experimental (Tabla 4) indicó que el $\mathrm{pH}$ fue el único factor que tuvo un efecto significativo sobre el índice de Langelier del agua remineralizada, ya que su valor-P (0.0026) fue menor al valor de la significancia prefijada ( $a=$ 0.05). El coeficiente de determinación R-cuadrada fue de $99.69 \%$; siendo superior al $70 \%$ recomendado para que el modelo interprete de forma adecuada los resultados.

Tabla 4. Análisis de varianza para el índice de Langelier.

\begin{tabular}{|l|c|c|c|c|c|}
\hline Parámetro & $\begin{array}{c}\text { Suma de } \\
\text { cuadrados }\end{array}$ & $\begin{array}{c}\text { Grados } \\
\text { de } \\
\text { libertad }\end{array}$ & $\begin{array}{c}\text { Cuadrado } \\
\text { medio }\end{array}$ & F & P \\
\hline A: $\mathrm{pH}$ & 4.6208 & 1 & 4.6208 & 86.34 & 0.0026 \\
\hline $\begin{array}{l}\text { B: tiempo de } \\
\text { residencia } \\
\text { (min) }\end{array}$ & 0.0128 & 1 & 0.0128 & 0.24 & 0.6583 \\
\hline
\end{tabular}


2020, Instituto Mexicano de Tecnología del Agua

Tecnología y

Ciencias $\cong$ Agua
Open Access bajo la licencia CC BY-NC-SA 4.0 (https://creativecommons.org/licenses/by-nc$\mathrm{sa} / 4.0 /$ )

\begin{tabular}{|l|c|c|c|c|c|}
\hline $\begin{array}{l}\text { C: velocidad } \\
\left(\mathrm{m} \mathrm{h}^{-1}\right)\end{array}$ & 0.0288 & 1 & 0.0288 & 0.54 & 0.5163 \\
\hline AB & 0.005 & 1 & 0.005 & 0.09 & 0.7799 \\
\hline AC & 0.0242 & 1 & 0.0242 & 0.45 & 0.5495 \\
\hline BC & 0.0002 & 1 & 0.0002 & 0 & 0.9551 \\
\hline Error & 0.16056 & 3 & 0.05352 & --- & --- \\
\hline Total & 4.85236 & 9 & --- & --- & --- \\
\hline
\end{tabular}

El diagrama de Pareto (Figura 3) permitió visualizar el impacto de cada uno de los factores, así como de las interacciones entre éstos en la variable de respuesta (índice de Langelier). En esta gráfica, cada efecto se divide entre su error estándar. En adición, se dibuja una línea sobre la gráfica para determinar si un efecto es estadísticamente significativo en el nivel de significancia especificado.

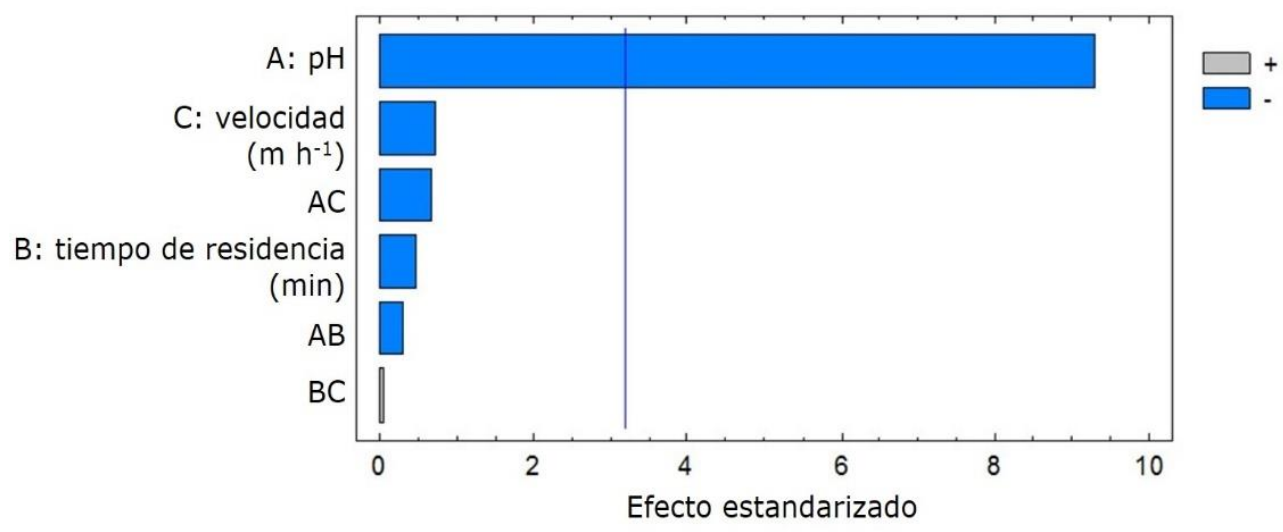

Figura 3. Diagrama de Pareto del diseño experimental. 


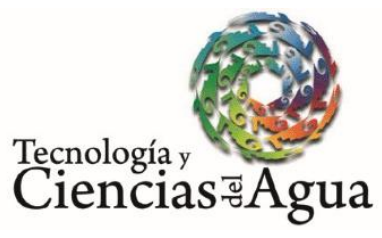

2020, Instituto Mexicano de Tecnología del Agua

Open Access bajo la licencia CC BY-NC-SA 4.0 (https://creativecommons.org/licenses/by-ncsa/4.0/)

Los factores y sus interacciones se grafican en orden descendente con relación al impacto con que afectan al índice de Langelier. El diagrama de Pareto muestra, al igual que el análisis de varianza, que sólo el pH tiene efecto significativo sobre el índice de estabilización de Langelier, pues es el único factor que cruza la línea de significancia. El diagrama de Pareto también mostró que el efecto es negativo, es decir, un valor menor de $\mathrm{pH}$ en el influente provoca obtener mayor ISL en el agua tratada.

Los gráficos de superficie de respuesta $\mathrm{pH}$-tiempo de residencia (Figura 4) y pH-velocidad (Figura 5) mostraron que al ajustar el influente a remineralizar a un $\mathrm{pH}$ de 3 se obtienen índices de Langelier mayores a $0 y \leq 0.5$, por lo que se obtiene un efluente con una estabilidad química aceptable, que no provocará problemas de corrosión o incrustación en la red de suministro. Los gráficos muestran que el ISL no se modificará si se varía el tiempo de residencia o la velocidad de flujo bajo las condiciones empleadas en este estudio, lo que es coherente con el ANOVA.

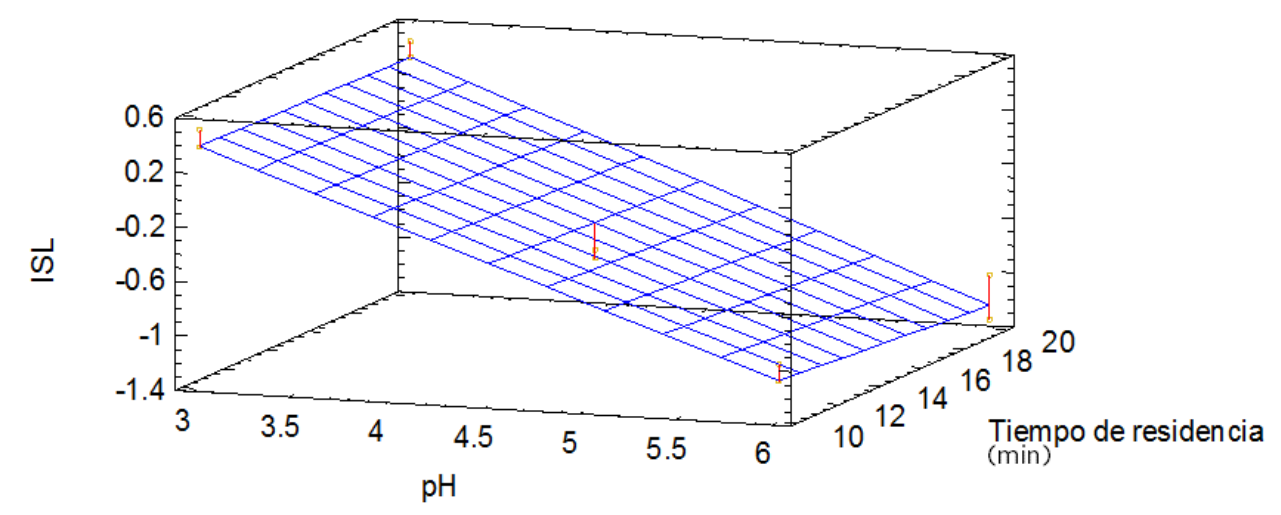

Figura 4. Gráfico de superficie de respuesta pH-tiempo de residencia. 


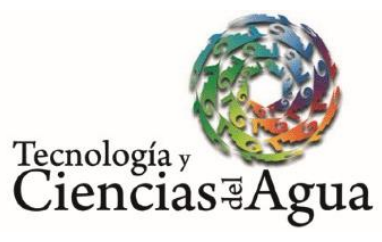

2020, Instituto Mexicano de Tecnología del Agua

Open Access bajo la licencia CC BY-NC-SA 4.0 (https://creativecommons.org/licenses/by-ncsa/4.0/)

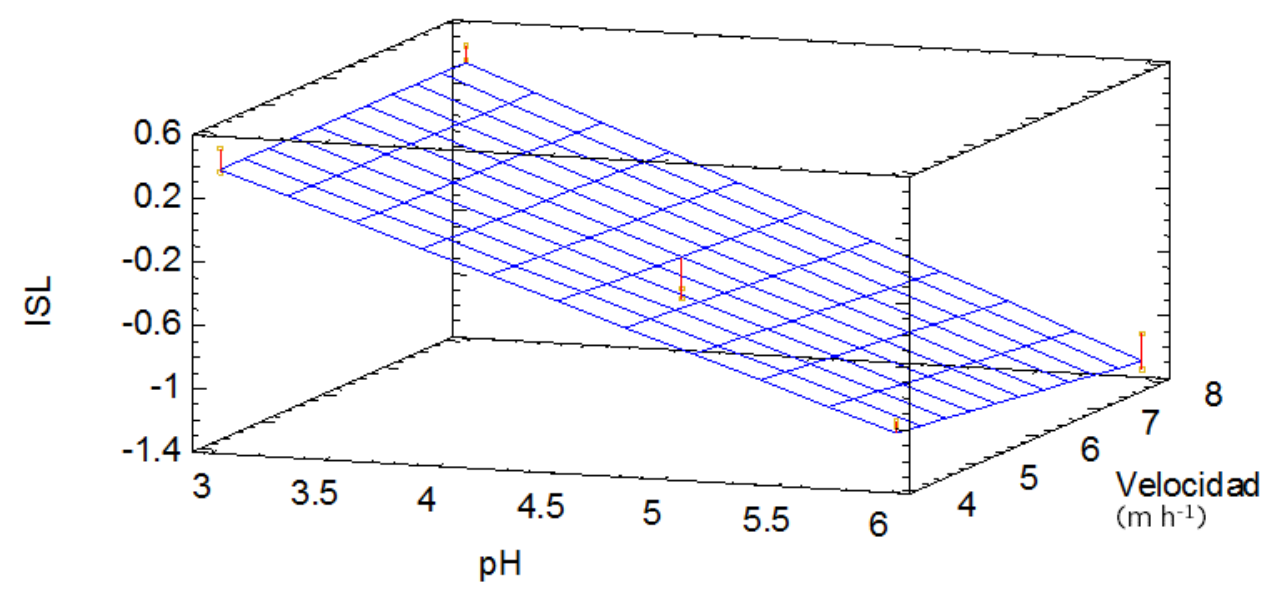

Figura 5. Gráfico de superficie de respuesta pH-velocidad.

\section{Etapa 2}

Una vez concluida la etapa 1 , se realizó un experimento bajo las mejores condiciones obtenidas, esto es: $\mathrm{pH}=3$, velocidad de flujo $=8\left(\mathrm{~m} \mathrm{~h}^{-1}\right)$ y tiempo de residencia $=10$ minutos. Las pruebas que se realizaron a $\mathrm{pH}$ $=3$ fueron las únicas que permitieron obtener valores de ISL $(0.4 \pm 0.1)$ que cumplen con el rango aceptable para agua remineralizada (ISL \pm 0.5 ) (Hernández-Suárez, 2010). Por otra parte, aunque se encontró que la velocidad de flujo y el tiempo de contacto no inciden en el ISL obtenido, estos parámetros se fijaron en el nivel alto y bajo, respectivamente, ya que tales condiciones permiten diseñar los contactores más pequeños $y$, Tecnología y ciencias del agua, ISSN 2007-2422,11(3), 277-308. DOI: 10.24850/j-tyca-202003-08 
Tecnología y

Ciencias: ־ Agua
2020, Instituto Mexicano de Tecnología del Agua

Open Access bajo la licencia CC BY-NC-SA 4.0 (https://creativecommons.org/licenses/by-nc$\mathrm{sa} / 4.0 /$ )

por lo tanto, requieren de menor costo de inversión. La duración de la prueba fue de 40 horas, realizando muestreos para analizar la calidad del agua cada dos horas.

La Figura 6 muestra el ISL obtenido durante la prueba en función del tiempo de operación. El proceso se comportó de manera estable, obteniendo un valor de ISL promedio de $0.467 \pm 0.034$, con una confianza de $95 \%$. El mínimo valor obtenido fue 0.33 , mientras que el máximo fue 0.56. Es decir, durante todo el tiempo que el sistema operó, se alcanzó un ISL aceptable.

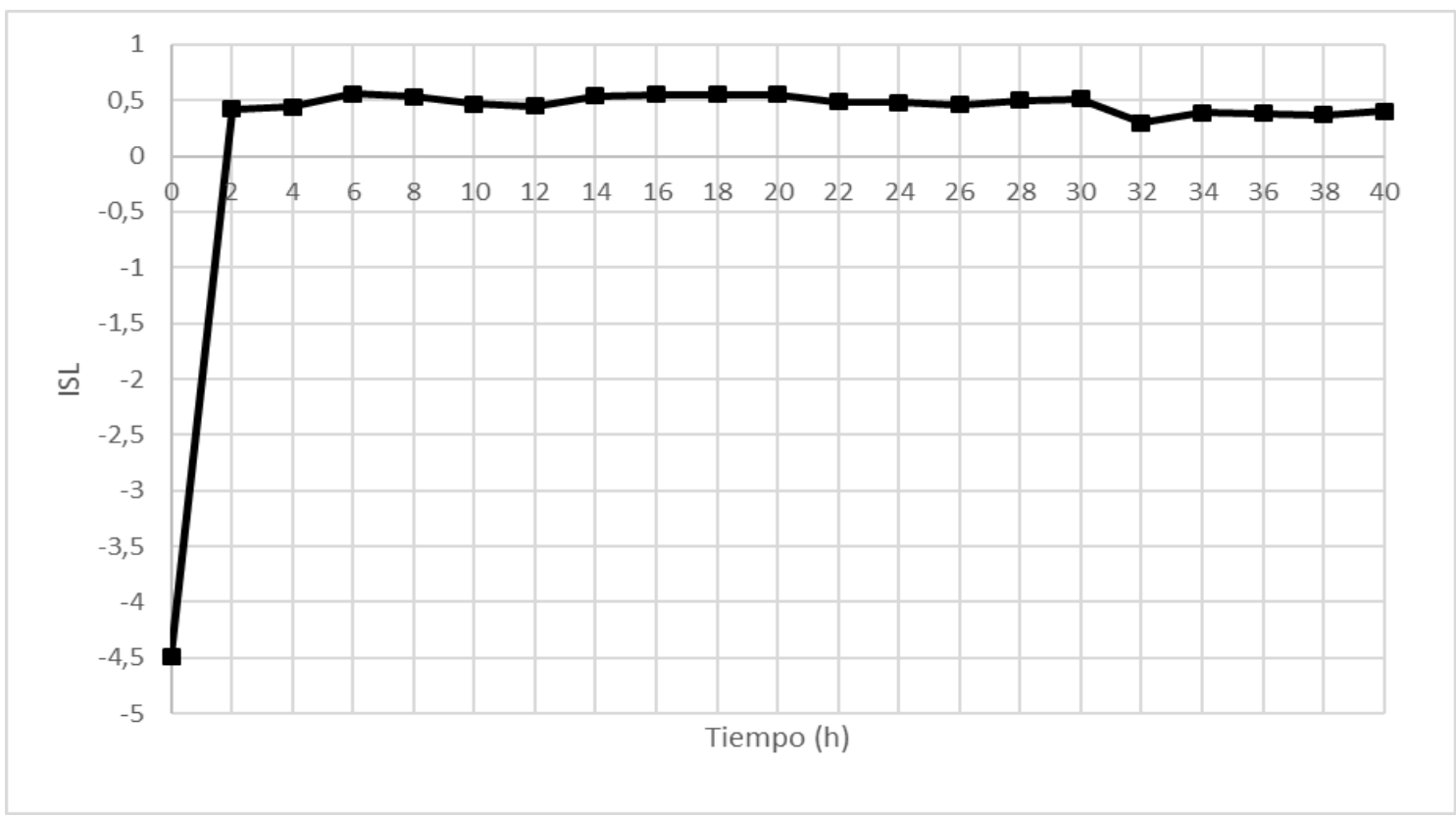

Figura 6. ISL en función del tiempo de operación.

La Tabla 5 muestra los resultados obtenidos de la calidad del agua osmotizada y del agua remineralizada, y se compara con los límites 


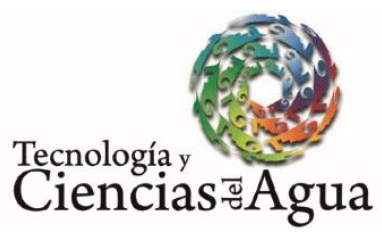

2020, Instituto Mexicano de Tecnología del Agua

Open Access bajo la licencia CC BY-NC-SA 4.0 (https://creativecommons.org/licenses/by-nc$\mathrm{sa} / 4.0 /$ )

establecidos por las regulaciones mexicana e israelí. El agua que se utilizó para el estudio (agua osmotizada) presentó muy baja concentración de dureza total y alcalinidad $\left(3.17 \pm 0.38 \mathrm{mg} \mathrm{l}^{-1} \mathrm{CaCO}_{3}\right.$ y $3.0 \pm 2.48 \mathrm{mg} \mathrm{L}^{-1}$ $\mathrm{CaCO}_{3}$, respectivamente); asimismo, presenta un ISL de $-4.58 \pm 0.22$. Estos valores no cumplen con los límites establecidos en la legislación israelí; sin embargo, con respecto a la normatividad mexicana (modificación del año 2000 a la NOM-127-SSA1-1994) (DOF, 1994), al no incluir los parámetros de alcalinidad, calcio e ISL, y al no establecer un límite mínimo permisible para la dureza total, el único parámetro con el que incumple la normatividad es el $\mathrm{pH}$, ya que es ligeramente inferior a $6.5(6.17 \pm 0.28)$. Este problema se resuelve por lo general en las plantas desalinizadoras mexicanas adicionándole un poco de sosa cáustica, para elevar el $\mathrm{pH}$ ( $\geq 6.5 \mathrm{y} \leq 8.5$ ); una vez que esto sucede, el agua cumple con los límites establecidos en la legislación mexicana y puede ser enviada a la red de distribución. Sin embargo, la ausencia de minerales provoca daños a la salud y al sistema de distribución de agua potable. Así, se debe contar con una normatividad más estricta en México, que incluya rangos para contenido mineral.

Tabla 5. Calidad del agua osmotizada y remineralizada. 
2020, Instituto Mexicano de Tecnología del Agua

Tecnología y

Ciencias $\approx$ Ag
Open Access bajo la licencia CC BY-NC-SA 4.0 (https://creativecommons.org/licenses/by-nc$\mathrm{sa} / 4.0 /$ )

\begin{tabular}{|c|c|c|c|c|}
\hline Parámetro & $\begin{array}{c}\text { Agua } \\
\text { osmotizada }\end{array}$ & $\begin{array}{c}\text { Agua } \\
\text { remine- } \\
\text { ralizada }\end{array}$ & $\begin{array}{c}\text { Regulación } \\
\text { israelí }\end{array}$ & $\begin{array}{c}\text { Regulación } \\
\text { mexicana }\end{array}$ \\
\hline $\mathrm{pH}$ & $6.17 \pm 0.28$ & $8.17 \pm 0.02$ & $7.5-8.3$ & $6.5-8.5$ \\
\hline $\begin{array}{c}\text { Alcalinidad } \\
\left(\mathrm{mg} \mathrm{L}^{-1}\right. \\
\left.\mathrm{CaCO}_{3}\right)\end{array}$ & $3.0 \pm 2.48$ & $83.2 \pm 2.54$ & $>80$ & $\mathrm{NE}$ \\
\hline \begin{tabular}{c}
$\mathrm{Calcio}\left(\mathrm{mg}_{-1}^{\left.\mathrm{CaCO}_{3}\right)}\right.$ \\
\hline $\left.\begin{array}{c}\text { Dureza total } \\
\left(\mathrm{mg} \mathrm{L}^{-1}\right. \\
\mathrm{CaCO}\end{array}\right)$
\end{tabular} & $3.17 \pm 0.38$ & $166.3 \pm 4.33$ & $160-240$ & $<500$ \\
\hline $\begin{array}{c}\text { Turbiedad } \\
(\mathrm{UTN})\end{array}$ & $0.317 \pm 0.19$ & $0.36 \pm 0.04$ & $<0.5$ & $<5$ \\
\hline \begin{tabular}{c} 
ISL \\
\hline
\end{tabular} & $-4.58 \pm 0.22$ & $0.47 \pm 0.03$ & $>0$ & $\mathrm{NE}$ \\
\hline
\end{tabular}

NE: no establecido.

Una vez remineralizada el agua osmotizada, se logró cumplir con los límites de los parámetros establecidos en la normatividad israelí para contenido mineral en agua de mar potabilizada. El tratamiento también permitió cumplir con la normatividad mexicana para los parámetros antes mencionados y mostrados en la Tabla 5. Aunque la normatividad mexicana no establece límites permisibles para ISL, concentración de calcio y alcalinidad, el proceso de remineralización no incrementó de 
Tecnología y

Ciencias $\approx$ Ag
2020, Instituto Mexicano de Tecnología del Agua

Open Access bajo la licencia CC BY-NC-SA 4.0 (https://creativecommons.org/licenses/by-nc-

$\mathrm{sa} / 4.0 /$ )

manera significativa la turbiedad ni provocó que se superara el límite máximo permisible de dureza. El pH agua remineralizada fue de $8.17 \pm$ 0.02, por lo que se mantuvo dentro del rango establecido en la Norma Oficial Mexicana. En contraste, Birnhack et al. (2011) reportan que una vez que el agua ha sido remineralizada con $\mathrm{CaCO}_{3}$ y $\mathrm{H}_{2} \mathrm{SO}_{4} \circ \mathrm{CO}_{2}$, es necesaria la adición de un reactivo químico o la eliminación del $\mathrm{CO}_{2}$ (Dreizin, Tenne, \& Hoffman, 2008; Migliorini \& Meinardi, 2005) para neutralizarel $\mathrm{pH}$.

Los resultados obtenidos en ambas etapas permitieron obtener los criterios de diseño para la construcción de contactores de calcita (Tabla 6), los cuales se encuentran dentro de los parámetros citados por Voutchkov (2011) y Hasson et al. (2017).

Tabla 6. Criterios para diseñar contactores de calcita.

\begin{tabular}{|l|c|c|}
\hline \multicolumn{1}{|c|}{ Parámetro } & Valor & Unidades \\
\hline $\begin{array}{l}\text { Velocidad máxima de flujo de agua en } \\
\text { contactor }\end{array}$ & 8 & $\mathrm{~m} \mathrm{~h}^{-1}$ \\
\hline $\begin{array}{l}\text { Tiempo de contacto en el lecho de } \\
\text { carbonato de calcio }\end{array}$ & 10 & $\mathrm{~min}$ \\
\hline Altura del lecho de calcita & 1.5 & $\mathrm{~m}$ \\
\hline Altura de capa de grava & 0.15 & $\mathrm{~m}$ \\
\hline $\begin{array}{l}\text { Dosis de ácido sulfúrico (al 98\%, grado } \\
\text { industrial) }\end{array}$ & 50 & $\mathrm{~mL} \mathrm{~m}^{-3}$ \\
\hline pH del agua a tratar & 3.5 & unidades \\
\hline
\end{tabular}


2020, Instituto Mexicano de Tecnología del Agua

Tecnología y

Ciencias $₫$ Agua
Open Access bajo la licencia CC BY-NC-SA 4.0 (https://creativecommons.org/licenses/by-nc$\mathrm{sa} / 4.0 /$ )

\begin{tabular}{|l|c|c|}
\hline $\begin{array}{l}\text { Tamaño efectivo del carbonato de } \\
\text { calcio empleado }\end{array}$ & 1 & $\mathrm{~mm}$ \\
\hline $\begin{array}{l}\text { Coeficiente de uniformidad del } \\
\text { carbonato de calcio }\end{array}$ & 1.29 & adimensional \\
\hline $\begin{array}{l}\text { Pureza del carbonato de calcio } \\
\text { empleado }\end{array}$ & $>98$ & $\%$ \\
\hline Consumo de carbonato de calcio & 0.163 & $\mathrm{~kg} \mathrm{~m}^{-3}$ \\
\hline
\end{tabular}

El costo de operación del proceso de remineralización se calculó en $\$ 0.04$ USD $\mathrm{m}^{-3}$, del cual $\$ 0.028$ USD $\mathrm{m}^{-3}$ corresponden a la dosificación de ácido sulfúrico y $\$ 0.012$ USD $\mathrm{m}^{-3}$ a la adición de carbonato de calcio (calcita).

Voutchkov (2011) presentó un desglose del costo de inversión y de operación y mantenimiento (O\&M) al remineralizar el agua de mar desalinizada por ósmosis inversa de una planta hipotética, con una capacidad de $100000 \mathrm{~m}^{3} \mathrm{~d}^{-1}$ mediante la adición de cal y $\mathrm{CO}_{2}$. El costo total para la producción de agua remineralizada fue de $0.058 \mathrm{USD} \mathrm{m}^{-3}$, lo cual correspondió a 0.016 USD $\mathrm{m}^{-3}$ de inversión, y 0.042 USD $\mathrm{m}^{-3}$ como resultado de la operación y mantenimiento. Este costo representa de 3 a $6 \%$ del costo total de producción de agua desalinizada mediante ósmosis inversa. Por otra parte, El-Azhar et al. (2012) reportaron costos de operación de 0.088 USD $\mathrm{m}^{-3}$ al utilizar la misma técnica (adición de cal y $\mathrm{CO}_{2}$ ). El influente provino de una planta desalinizadora ubicada en Marruecos, la cual cuenta con una capacidad de $13000 \mathrm{~m}^{3} \mathrm{~d}^{-1}$. 


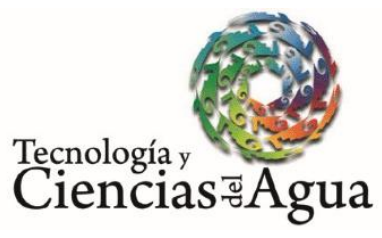

2020, Instituto Mexicano de Tecnología del Agua

Open Access bajo la licencia CC BY-NC-SA 4.0 (https://creativecommons.org/licenses/by-nc$\mathrm{sa} / 4.0 /$ )

Es importante mencionar que un inconveniente que se presenta al utilizar esta técnica es que la calcita se va disolviendo y eventualmente debe ser repuesta. Esto, en la práctica, puede dificultar la operación del contactor si no es diseñado de manera correcta para reabastecerse de calcita con la menor frecuencia posible. Asimismo, el contactor debe contar con elementos que permitan su reabastecimiento con relativa facilidad: mirilla transparente para observar el nivel de calcita en el contactor; registro tipo hombre en la parte superior del contactor, para reponer la calcita que se ha disuelto; escalera y pasillo en la parte superior, para permitir al operador subir y reponer la calcita con seguridad.

A manera de ejemplo, para un contactor que remineralice un caudal de $10 \mathrm{~L} \mathrm{~s}^{-1}$ (864 $\mathrm{m}^{3} \mathrm{~d}^{-1}$ ), bajo los criterios de diseño y consumo de reactivos obtenidos en el presente estudio, y mostrados en la Tabla 6, el consumo de calcita sería de 4.25 toneladas por mes, y si el diámetro del contactor se establece de $2 \mathrm{~m}$, se podría llenar con $1.5 \mathrm{~m}$ de calcita. Bajo este escenario, se presentará una reducción de altura de $0.3 \mathrm{~m}$ de calcita cada mes. La reposición se podría realizar hasta que el nivel de calcita sea de $0.5 \mathrm{~m}$, sin provocar variación en la calidad de agua remineralizada, pues la calidad del agua depende sólo del pH del agua que ingresa al contactor, y no del tiempo de residencia o la velocidad. La reposición de calcita se podría realizar cada tres meses aproximadamente. 


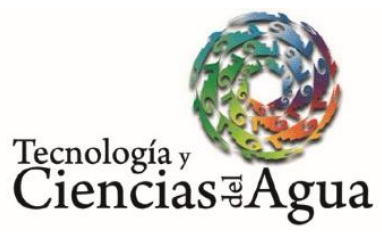

2020, Instituto Mexicano de Tecnología del Agua

Open Access bajo la licencia CC BY-NC-SA 4.0 (https://creativecommons.org/licenses/by-nc$\mathrm{sa} / 4.0 /$ )

\section{Conclusiones}

De los tres factores evaluados en este estudio $(\mathrm{pH}$ del influente, tiempo de residencia y velocidad de flujo), sólo el pH del influente tuvo un efecto estadísticamente significativo en el índice de saturación de Langelier durante el proceso de remineralización utilizando un contactor de calcita a flujo continuo. El pH presentó un efecto inverso sobre el ISL, es decir, el menor valor de $\mathrm{pH}$ en el influente del proceso provocó el mayor incremento en el ISL del agua remineralizada, siendo el $\mathrm{pH}$ de tres el valor óptimo establecido. Es posible diseñar contactores de calcita con una velocidad de flujo de $8 \mathrm{~m} \mathrm{~h}^{-1}$, y un tiempo de residencia hidráulico de 10 min (que implican la construcción de contactores más compactos), y por lo tanto resulta en un menor costo de inversión, en comparación con contactores que operen a una velocidad de hasta $4 \mathrm{~m} \mathrm{~h}^{-1}$ y tiempos de residencia de 30 minutos; esto, sin afectar la eficiencia de remineralización. Durante el presente estudio fue posible obtener agua remineralizada con un índice de Langelier de $0.47 \pm 0.03$, es decir, sin tendencia a provocar problemas de corrosión o incrustación. La calidad del agua remineralizada cumplió con los límites establecidos para el contenido mineral en las regulaciones israelí y mexicana para agua potable. El costo de operación del proceso se calculó en $\$ 0.04$ USD $\mathrm{m}^{-3}$, el cual es menor que el de otras técnicas reportadas. 


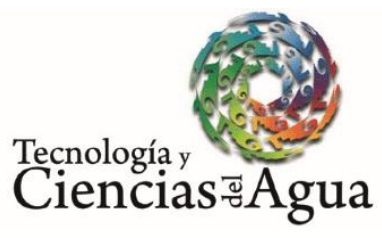

2020, Instituto Mexicano de Tecnología del Agua

Open Access bajo la licencia CC BY-NC-SA 4.0 (https://creativecommons.org/licenses/by-ncsa/4.0/)

\section{Referencias}

Awatif, A., Basim, K., \& Sura-Ali, K. (2014). Evaluating water stability indices from water treatment plants in Baghdad city. Water Resource and Protection, 2(1351), 1-10.

Bergman, R. (2007). Reverse osmosis and nanofiltration: Manual of water supply practices-M46, $2^{\text {nd }}$ ed. Denver, USA: American Water Works Association.

Birnhack, L., Voutchkov, N., \& Lahav, O. (2011). Fundamental chemistry and engineering aspects of post-treatment processes for desalinated water- A review. Desalination, 273, 6-22.

Biyoune, M. G., Atbir, A., Bari, H., Hassnaoui, L., Mongach, E., Khadir, A., Boukbir, L., Bellajrou, R. , \& Elhadek, M. (2017). Remineralization of permeate water by calcite bed in the Daoura's plant (south of Morocco). European Physical Journal Special Topics, 226, 931-941.

Conagua, Comisión Nacional del Agua. (2015). Estadísticas del agua en México, edición 2015. Ciudad de México, México: Comisión Nacional del Agua.

Delion, N., Mauguin, G., \& Corsin, R. (2004). Importance and impact of post treatments on design and operation of SWRO plants. Desalination, 165, 323-334.

DOF, Diario Oficial de la Federación. (1994). Norma Oficial Mexicana. NOM-127-SSA1-1994. Salud ambiental, agua para uso y consumo humano. Límites permisibles de calidad y tratamientos a que debe someterse el agua para su potabilización. Diario Oficial de la Federación. México, DF, México. 
Tecnología y

Ciencias: ־ Agua
2020, Instituto Mexicano de Tecnología del Agua

Open Access bajo la licencia CC BY-NC-SA 4.0 (https://creativecommons.org/licenses/by-nc$\mathrm{sa} / 4.0 /$ )

Dreizin, Y., Tenne, A., \& Hoffman, D. (2008). Integrating large scale seawater desalination plants within Israel's water supply system. Desalination, 220, 132-149.

Edwards, M. (2004). Controlling corrosion in drinking water distribution systems: A grand challenge for the 21st century. Water Science and Technology, 49(2), 1-8.

El-Azhar, F., Tahaikt, M., Zouhri, N., Zdeg, A., Hafsi, M., Tahri, K., Bari, H., Taky, M., Elamrani, M., \& Elmidaoui, A. (2012). Remineralization of Reverse-Osmosis (RO)-desalted water for a Moroccan desalination plant: Optimization and cost evaluation of the lime saturator post. Desalination, 30, 46-50.

Gutiérrez, P., \& Salazar, R. (2012). Análisis y diseño de experimentos, 3a ed. México, DF, México: McGraw-Hill.

Hernández-Suárez, M. (2010). Guía para la re-mineralización de las aguas desaladas, $2^{\mathrm{a}}$ ed. Las Palmas, España: Ministerio de Medio Ambiente y Medio Rural y Marino.

Hasson, D., Fine, L., Sagiv, A., Semiat, R. , \& Shemer, H. (2017). Modeling remineralization of desalinated water by micronized calcite dissolution. Environmental Science and Technology, 51, 1248112488.

Kozisek, F., Rosborg, I., Selinus, O., Ferrante, M. , \& Jovanovic, D. (2015). Background. In: Rosborg, I. (ed.). Drinking water minerals and mineral balance: Importance, Health Significance, Safety Precautions (pp. 1-23). Cham, Suiza: Springer. 


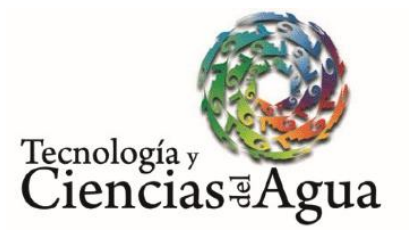

2020, Instituto Mexicano de Tecnología del Agua

Open Access bajo la licencia CC BY-NC-SA 4.0 (https://creativecommons.org/licenses/by-ncsa/4.0/)

Lehmann, O., Birnhack, L., \& Lahav, O. (2013). Design aspects of calcitedisolution reactors applied for post-treatment of desalinated water. Desalination, 314, 1-9.

Liang, J., Deng, A., Xie, R., Gomez, M., Hu, J., Zhang, J., Nam, C., \& Adin, A. (2013a). Impact of seawater reverse osmosis (SWRO) product remineralization on the corrosion rate of water distribution pipeline materials. Desalination, 311, 54-61.

Liang, J., Deng, A., Xie, R., Gomez, M., Hu, J., Zhang, J., Nam, C., \& Adin, A. (2013b). Impact of flow rate on corrosion of cast iron and quality of re-mineralized seawater reverse osmosis (SWRO) membrane product water. Desalination, 322, 76-83.

McNeill, L., \& Edwards, M. (2001). Iron pipe corrosion in distribution systems. Journal American Water Works Association, 93(7), 88100.

Migliorini, G., \& Meinardi, R. (2005). 40 MIGD potabilization plant at Ras Laffan: design and operating experience. Desalination, 182, 275282.

Pauta-Novillo, A. D., \& Quezada-Sisalima, D. A. (2017). Determinación de la tendencia corrosiva e incrustante del agua potable distribuida en la ciudad de Azogues (tesis de licenciatura). Universidad de Cuenca, Cuenca, Ecuador.

Sarin, P., Snoeyink, V. L., Bebee, J., Kriven, W., \& Clement, J. (2001). Physico-chemical characteristics of corrosion scales in old iron pipes. Water Research, 35 (12), 2961-2969. 


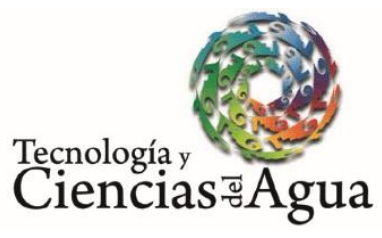

2020, Instituto Mexicano de Tecnología del Agua

Open Access bajo la licencia CC BY-NC-SA 4.0 (https://creativecommons.org/licenses/by-nc$\mathrm{sa} / 4.0 /$ )

Shemer, H., Hasson, D., \& Semiat, R. (2015). State-of-the-art review on post-treatment technologies. Desalination, 356, 285-293.

Sousi, M., Liu, G., Salinas-Rodríguez, S. G., Knezev, A., Blankert, B., Schippers, J. C., Van-Der-Merr, W., \& Kennedy, M. (2018). Further developing the bacterial growth potential method for ultra-pure drinking water produced by remineralization of reverse osmosis permeate. Water Research, 145, 687-696.

Tavanpour, N., Noshadi, M., \& Navid, T. (2016). Scale formation and corrosion of drinking water pipes: A case study of drinking water distribution system of Shiraz city. Modern Applied Science, 10(3), $1-12$.

Vasiliy, V., Garbera, O., \& Chenb, Y. (2018). Magnesium deficiency in tap water in Israel: The desalination era. Desalination, 426, 88-96.

Vingerhoeds, M., Nijenhuis-De-Vries, M., Ruepert, N., Van-Der-Laan, H., Bredie, W., \& Kremer, S. (2016). Sensory quality of drinking water produced by reverse osmosis membrane filtration followed by remineralisation. Water Research, 94, 42-51.

Voutchkov, N. (2011). Remineralization of desalinated water. A SunCam online continuing education course. Recuperado de https://s3.amazonaws.com/suncam/docs/118.pdf?1517305879

Withers, A. (2005). Options for recarbonation, remineralization and disinfection for desalination plants. Desalination, 179, 11-24. 